\title{
Structure of the Spectra and Resonances of Schrödinger Operators
}

\section{Tahar Bouguetaia ${ }^{1}$ and Bekkai Messirdi ${ }^{2 *}$}

High School of Electrical and Energetic Engineering, ESGEE-Oran, Laboratory of Fundamental and Applicable Mathematics of Oran (LMFAO), University of Oran, Ahmed Ben Bella, Algeria ("bmessirdi@yahoo.fr).

\begin{abstract}
The main goal of this paper is to study the spectrum and resonances of several classes of Schrödinger operators. Two important examples occurring in mathematical physics are discussed: harmonic oscillator and Hamiltonian of hydrogen atom.
\end{abstract}

Keywords: Schrödinger operator, Spectrum, Periodic potential, Resonances.

AMS Mathematics subjectif classification (2000): 47A10, 35J10, $35 \mathrm{~B} 34$.

\section{INTRODUCTION}

A big problem in mathematical physics is the description of spectral properties of Schrödinger operators. Here we present some fundamental theorems about spectral and resonance theory of Schrödinger operators. We look at some important examples occurring in mathematical physics: the harmonic oscillator and the hydrogen atom. The spectrum of Laplace operator, $\Delta=$ $\sum_{j=1}^{n} \frac{\partial^{2}}{\partial x_{j}^{2}}$ on the space $L^{2}\left(\mathbb{R}^{n}\right)$ of square-integrable functions on $\mathbb{R}^{n}$ (the state space in quantum mechanics), has many applications in geometry, topology, physics, chemistry etc. We define the Schrödinger operator on the manifold $\mathbb{R}^{n}$ by the linear unbounded operator $P=-\Delta+V$, on the set of smooth compact supports functions $C_{c}^{\infty}\left(\mathbb{R}^{n}\right) \subset L^{2}\left(\mathbb{R}^{n}\right)$, where $V: \mathbb{R}^{n} \rightarrow \mathbb{R}$ is the multiplication operator associated with the potential $V(x)$ in the Hilbert space $L^{2}\left(\mathbb{R}^{n}\right)$ equipped with the inner product $\langle\varphi, \psi\rangle=\int_{\mathbb{R}^{n}} \varphi(x) \overline{\psi(x)} d x$, and the associated norm

$$
\|\varphi\|=\sqrt{\int_{\mathbb{R}^{n}}|\varphi(x)|^{2} d x} ; \varphi, \psi \in L^{2}\left(\mathbb{R}^{n}\right) .
$$

All the information about a quantum mechanical system (atoms, molecules, nuclei, solids, etc...) is contained in the Schrödinger operator for the system and in particular in the structure of its spectrum. In many applications, $V$ is $-\Delta$-bounded with bound $<1$. In this case, we may apply the Kato-Rellich theorem to deduce that $P$ is self-adjoint on domain $D(P)=$ $H^{2}\left(\mathbb{R}^{n}\right)$, where $H^{2}\left(\mathbb{R}^{n}\right)=\left\{\varphi \in L^{2}\left(\mathbb{R}^{n}\right): D^{\alpha} \varphi \in L^{2}\left(\mathbb{R}^{n}\right), \alpha \in \mathbb{N}^{n},|\alpha| \leq 2\right\}$ and $D^{\alpha} \varphi$ are the weak derivatives of $\varphi$.

Present paper is devoted to the spectral analysis of Schrödinger operators acting on $L^{2}\left(\mathbb{R}^{n}\right), n \geq 1$, where, we present a detailed mathematical study of the spectra and resonances 
of the operator $P$, which is both accessible to theoretical physicists and chemists and also to researchers in the field of applied mathematics. The essential and discrete spectrum and resonances for such operators have been the subject of a vast literature over the last 20 years, a detailed review and references are found in the book series of Reed and Simon (Volume 3, 1979 and Volume 4, 1978) and publications (Aguilar and Combes, 1971; Balslev and Combes, 1971; Brüning, 1989; Muminov and Shodiev, 2011).

We define the discrete spectrum $\sigma_{\text {disc }}(A)$ of a self-adjoint operator $A$ as the set of eigenvalues of $A$ having finite multiplicity and being isolated points of the spectrum. The essential spectrum of $A$ is the set $\sigma_{e s s}(A)=\sigma(A) \backslash \sigma_{\text {disc }}(A)$.

We thus have the disjoint decomposition $\sigma(A)=\sigma_{\text {disc }}(A) \cup \sigma_{e s s}(A)$. Obviously, $\sigma_{\text {ess }}(A)$ consists of all accumulation points of $\sigma(A)$ and all eigenvalues of infinite multiplicity. The essential spectrum of a self-adjoint operator is a closed subset of $\mathbb{R}$, it can be characterized as that part of the spectrum that is invariant under compact perturbations, this is the content of Weyl's Theorem, and so, for a Schrödinger operator should morally depend only on properties of the potential $V$.

We propose to present some fundamental theorems of Schrödinger operators and their spectral theory considering the following five situations:

(1) $V(x) \rightarrow \infty$ as $|x| \rightarrow \infty$;

(2) $V(x) \rightarrow 0$ as $|x| \rightarrow \infty$;

(3) $V: \mathbb{R}^{n} \rightarrow \mathbb{R}$ bounded and continuous;

(4) $V: \mathbb{R}^{n} \rightarrow \mathbb{R}$ periodic; and

(5) $V(x)=x . k, k \in \mathbb{R}^{n}$ (Stark-effect).

We also describe the resonances of $P$ by using the analytic dilation in a complex strip for suitable potentials (complex Scaling method). Resonances of $P$ are complex eigenvalues of the non-hermitian Hamiltonian obtained by complex scaling. Resonance theory of Schrödinger operators based on the complex scaling method is detailed in Messirdi (1994); and Messirdi et al. (2018). However, since the present problem is partly motivated by recent investigations of resonances in periodic structures, we have chosen to investigate resonances Schrödinger operators with periodic potentials.

Note that the problem of computing resonances of $P$ with $V$ periodic has been much less studied, it is an interesting physical problem with major mathematical difficulties. The resonances of $P$ are accessible by meromorphic extension of the resolvent $(P-z)^{-1}$, we 
explain the different steps necessary to get this extension from the upper half plane through the spectrum of $P$. The singularities of $(P-z)^{-1}$ are effectively the resonances of $P$.

The structure of this paper is as follows. First, in section 2 we study the spectral properties of $-\Delta$ on $L^{2}\left(\mathbb{R}^{n}\right)$. Section 3, is dedicated to a spectral study of Schrödinger operator $P$ in different physical situations where, $V(x) \rightarrow \infty ; V(x) \rightarrow 0$ as $|x| \rightarrow \infty ; V$ is bounded and continuous; $V$ is periodic and $V(x)=x . k, k \in \mathbb{R}^{n}$ (Stark-effect), respectively.

Another critical area of mathematical quantum mechanics lies in finding resonances of $P$ with a given potential is discussed in section 4 of this paper. We also investigate the situation where the potential $V$ is periodic and we compute explicitly the energy spectrum and resonances of hydrogen atom.

\section{SPECTRAL PROPERTIES OF FREE SCHRÖDINGER OPERATOR}

Let us first study the spectral properties of $-\Delta$ on $L^{2}\left(\mathbb{R}^{n}\right)$. We will see later that $-\Delta$ does not have eigenvalues. Indeed, the spectrum of $-\Delta$ is purely essential covering positive half line.

2.1. Proposition: $\sigma_{e s s}(-\Delta)=[0,+\infty[$.

Proof: According to the integration by parts formula, we have:

$\langle-\Delta \varphi, \varphi\rangle=\int_{\mathbb{R}^{n}}(-\Delta \varphi)(x) \overline{\varphi(x)} d x=\int_{\mathbb{R}^{n}}|\nabla \varphi(x)|^{2} d x$, for all $\varphi \in C_{c}^{\infty}\left(\mathbb{R}^{n}\right)$.

Given, $\varphi \in H^{2}\left(\mathbb{R}^{n}\right)$, there exists a sequence $\left(\varphi_{k}\right)_{k \in \mathbb{N}} \subset C_{c}^{\infty}\left(\mathbb{R}^{n}\right)$ such that $\varphi_{k} \rightarrow \varphi$ and $-\Delta \varphi_{k} \rightarrow-\Delta \varphi$ as $k \rightarrow \infty$, then $\langle-\Delta \varphi, \varphi\rangle \geq 0$ for all $\varphi \in H^{2}\left(\mathbb{R}^{n}\right)$.

In particular, $-\Delta$ is a positive self-adjoint operator on the domain $H^{2}\left(\mathbb{R}^{n}\right)$, so the spectral theorem shows that $\sigma(-\Delta) \subset[0,+\infty[$.

For the reverse inclusion, Weyl criterion is used characterizing the essential spectrum as follows:

$\lambda \in \sigma_{e s s}(-\Delta)$ if and only if there exists a sequence $\left(\varphi_{k}\right)_{k \in \mathbb{N}} \subset H^{2}\left(\mathbb{R}^{n}\right)$ such that $\left\|\varphi_{k}\right\|=1$ and for all $k \in \mathbb{N},\left(\varphi_{k}\right)_{k \in \mathbb{N}}$ is a weak null sequence, i.e. $\varphi_{k} \rightarrow 0$ weakly, and $\left\|(-\Delta-\lambda) \varphi_{k}\right\| \rightarrow 0$ (such a sequence is called a singular Weyl sequence of $-\Delta$ and $-\lambda$ ).

Let $\lambda \in\left[0,+\infty\left[, z \in \mathbb{R}^{n}\right.\right.$ so that $|z|=\sqrt{\lambda}$ and $\omega(x)=e^{i x . z}, x \in \mathbb{R}^{n}$.

Clearly, $\omega \notin H^{2}\left(\mathbb{R}^{n}\right)$, but we have point wise:

$-\Delta \omega(x)=\lambda \omega(x)$ for all $x \in \mathbb{R}^{n}$.

Let $B_{r}=\left\{x \in \mathbb{R}^{n}:|x|<r\right\}$ be the open ball with radius $r>0$ around zero in $\mathbb{R}^{n}$. Then, there is a cut-off function $\psi \in C_{c}^{\infty}\left(\mathbb{R}^{n}\right)$ with support in $B_{2}, 0 \leq \psi \leq 1$ and $\psi=1$ on $B_{1}$. 
Define $\psi_{k}(x)=\psi\left(\frac{x}{k}\right), x \in \mathbb{R}^{n}, k \in \mathbb{N}^{*}$, so that $\psi_{k}(x)=1$ for $|x| \leq k, \psi_{k}(x)=0$ for $|x| \geq 2 k$ and $\left|\nabla \psi_{k}(x)\right| \leq M k^{-1},\left|\frac{\partial^{2} \psi_{k}}{\partial x_{i} \partial x_{j}}\right| \leq M k^{-2}$, with a suitable constant $M>0$.

We set $\eta_{k}(x)=\psi_{k}(x) \omega(x)$ and $\varphi_{k}(x)=\frac{\eta_{k(x)}}{\left\|\eta_{k}\right\|}, k \in \mathbb{N}^{*}$. It is clear that $\left\|\varphi_{k}\right\|=1$, also show that $\varphi_{k} \rightarrow 0$ weakly in $L^{2}\left(\mathbb{R}^{n}\right)$. Let $\varphi \in C_{c}^{\infty}\left(\mathbb{R}^{n}\right)$, write $\Omega_{\mathrm{k}}=\mathrm{B}_{2 \mathrm{k}} \backslash \mathrm{B}_{\mathrm{k}}$ and observe that:

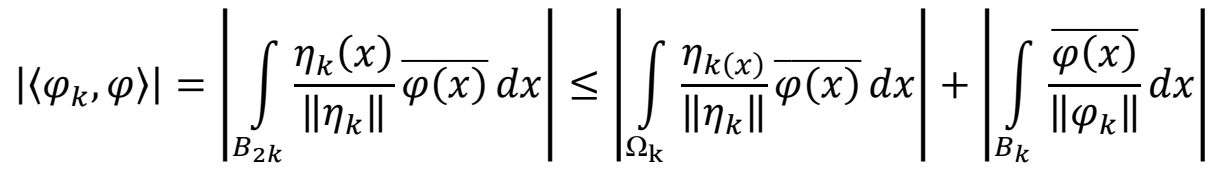

$$
\begin{aligned}
& \leq \frac{\|\varphi\|_{L^{1}\left(\mathbb{R}^{n}\right)}}{\left\|\eta_{k}\right\|}+\left|\int_{\Omega_{\mathrm{k}}} \frac{\eta_{k}(x)}{\left\|\eta_{k}\right\|} \overline{\varphi(x)} d x\right|
\end{aligned}
$$

where, $\|\varphi\|_{L^{1}\left(\mathbb{R}^{n}\right)}=\int_{\mathbb{R}^{n}}|\varphi(x)| d x$.

However, ||$\eta_{k}||^{2}=\int_{\mathbb{R}^{n}}\left|\psi_{k}(x) \omega(x)\right|^{2} d x \geq \int_{B_{k}}|\omega(x)|^{2} d x=\left|B_{k}\right| \underset{k \rightarrow \infty}{\longrightarrow} \infty$ and that the second term on the right-hand side of (2.2) vanishes for $k \geq K$ and when the support of $\varphi$ is included in $B_{K}$. Thus, $\left|\left\langle\varphi_{k}, \varphi\right\rangle\right| \underset{k \rightarrow \infty}{\longrightarrow} 0$. As $C_{c}^{\infty}\left(\mathbb{R}^{n}\right)$ is dense in $L^{2}\left(\mathbb{R}^{n}\right)$, we conclude that $\left|\left\langle\varphi_{k}, \varphi\right\rangle\right| \underset{k \rightarrow \infty}{\longrightarrow} 0$ for any $\varphi \in L^{2}\left(\mathbb{R}^{n}\right)$.

Then, check that $\left\|(-\Delta-\lambda) \varphi_{k}\right\| \rightarrow 0$ as $k \rightarrow \infty$.

For $\varphi \in C_{c}^{\infty}\left(\mathbb{R}^{n}\right)$, we have:

$$
\begin{aligned}
-\Delta\left(\psi_{k} \varphi\right) & =-\sum_{j=1}^{n} \frac{\partial^{2}}{\partial x_{J}^{2}}\left(\psi_{k} \varphi\right)=-\sum_{j=1}^{n}\left[\varphi \frac{\partial^{2} \psi_{k}}{\partial x_{j}^{2}}+2 \frac{\partial \psi_{k}}{\partial x_{j}} \frac{\partial \varphi}{\partial x_{j}}+\psi_{k} \frac{\partial^{2} \varphi}{\partial x_{j}^{2}}\right] \\
& =-\varphi \Delta \psi_{k}-2\left\langle\nabla \psi_{k}, \nabla \varphi\right\rangle-\psi_{k} \Delta \varphi,
\end{aligned}
$$

and $\quad\left\|(-\Delta-\lambda) \varphi_{k}\right\|^{2}=\int_{\mathbb{R}^{n}}\left|(-\Delta-\lambda) \frac{\eta_{k}(x)}{\left\|\eta_{k}\right\|}\right|^{2} d x$

$$
=\frac{1}{\left\|\eta_{k}\right\|^{2}}\left[\int_{B_{k}}\left|(-\Delta-\lambda) e^{i x . z}\right|^{2} d x+\int_{\Omega_{k}}\left|(-\Delta-\lambda)\left(\psi_{k}(x) \omega(x)\right)\right|^{2} d x\right] .
$$

The first term on the right-hand side of (2.4) vanishes according to (2.1). Using once again the identity (2.1) and equation (2.3), we get:

$$
\begin{aligned}
||(-\Delta & -\lambda) \varphi_{k} \|^{2}=\frac{1}{\left\|\eta_{k}\right\|^{2}} \int_{\Omega_{k}}\left|2\left\langle\nabla \psi_{k}, \nabla \omega\right\rangle+\omega \Delta \psi_{k}\right|^{2} d x \\
& \leq \frac{2}{\left\|\eta_{k}\right\|^{2}}\left[\int_{\Omega_{k}}\left|2\left\langle\nabla \psi_{k}, z\right\rangle\right|^{2} d x+\int_{\Omega_{k}}\left|\Delta \psi_{k}\right|^{2} d x\right] \\
& \leq \frac{2}{\left\|\eta_{k}\right\|^{2}}\left[4 \lambda \int_{\Omega_{k}}\left|\nabla \psi_{k}\right|^{2} d x+\int_{\Omega_{k}}\left|\Delta \psi_{k}\right|^{2} d x\right] .
\end{aligned}
$$


So, there exist positive constants $C_{1}$ and $C_{2}$ such that:

$$
\begin{aligned}
& \left\|(-\Delta-\lambda) \varphi_{k}\right\|^{2} \leq \frac{\left|\Omega_{\mathrm{k}}\right|}{\left|B_{k}\right|}\left(\frac{C_{1}}{k^{2}}+\frac{C_{2}}{k^{4}}\right)=\frac{(2 k)^{n}-k^{n}}{k^{n}}\left(\frac{C_{1}}{k^{2}}+\frac{C_{2}}{k^{4}}\right) \\
& =\left(2^{n}-1\right)\left(\frac{C_{1}}{k^{2}}+\frac{C_{2}}{k^{4}}\right) \rightarrow 0 \text { as } k \rightarrow \infty .
\end{aligned}
$$

It is concluded that the operator $-\Delta$ on $L^{2}\left(\mathbb{R}^{n}\right)$ with domain $H^{2}\left(\mathbb{R}^{n}\right)$ does not have eigenvalues. In fact, the spectrum of $-\Delta$ is purely continuous.

2.2. Proposition: If $\varphi \in H^{2}\left(\mathbb{R}^{n}\right)$ is such that $-\Delta \varphi=\lambda \varphi$ for some $\lambda \geq 0$, then $\varphi$ is infinitely differentiable on $\mathbb{R}^{n}$.

Proof: It follows from the equation $-\Delta \varphi=\lambda \varphi$ that if $\varphi \in H^{2}\left(\mathbb{R}^{n}\right)$, then $-\Delta \varphi \in H^{2}\left(\mathbb{R}^{n}\right)$ and so $\varphi \in H^{4}\left(\mathbb{R}^{n}\right)$. Iterating in this manner, it follows that $\varphi$ is in any positive indexed Sobolev space. So, the function $\varphi$ is infinitely differentiable.

\section{SPECTRAL PROBLEM BACKGROUND FOR SCHRÖDINGER OPERATOR}

For Schrödinger operators on $L^{2}\left(\mathbb{R}^{n}\right)$, consider $P$ as a perturbation of $-\Delta$ by the potential $V$ with domain $D(P)=H^{2}\left(\mathbb{R}^{n}\right) \cap\left\{\varphi \in L^{2}\left(\mathbb{R}^{n}\right): \varphi V \in L^{2}\left(\mathbb{R}^{n}\right)\right\}$.

Some important spectral results for Schrödinger operators are presented below. We first recall Persson formula for the infimum of the essential spectrum (Persson, 1960).

3.1. Theorem: Let $V$ be bounded operator on $L^{2}\left(\mathbb{R}^{n}\right)$, then:

$$
\inf \sigma_{e s s} P=\sup _{K \subset \mathbb{R}^{n},{ }_{K \text { compact }} \varphi \in C_{c}^{\infty}\left(\mathbb{R}^{n}, K\right),\|\varphi\|=1}\langle P \varphi, \varphi\rangle .
$$

Proof: (See Cycon et al., 1987).

Consider the cases: (1) $V(x) \rightarrow \infty$ as $|x| \rightarrow \infty$;

(2) $V(x) \rightarrow 0$ as $|x| \rightarrow \infty$;

(3) $V: \mathbb{R}^{n} \rightarrow \mathbb{R}$ bounded and continuous;

(4) $V: \mathbb{R}^{n} \rightarrow \mathbb{R}$ periodic; and

(5) $V(x)=x \cdot k, k \in \mathbb{R}^{n}$ (Stark-effect).

3.1. $\boldsymbol{V}(\boldsymbol{x}) \rightarrow \infty$ for $|\boldsymbol{x}| \rightarrow \infty$. Consider the operator $P=-\Delta+V$ defined on $L^{2}\left(\mathbb{R}^{n}\right)$ such that $V$ is locally bounded on $\mathbb{R}^{n}$, real valued function and $\lim _{|x| \rightarrow \infty} V(x)=+\infty$.

The most important example in this class is the anharmonic oscillator for which $V(x)=$ $|x|^{2 k}, k \in \mathbb{N}^{*}$. I denotes in the following the identity operator.

3.2. Lemma: If $V \geq 0$ is a non-negative potential and $W$ is multiplication by a bounded function of compact support, then $W$ is P-compact, i.e. $W(I+P)^{-1}$ is compact operator on $L^{2}\left(\mathbb{R}^{n}\right)$. 
Proof: Note first that $I+P$ is a positive operator, then $(I+P)^{-1}$ is bounded on $L^{2}\left(\mathbb{R}^{n}\right)$. On the other hand, $W(I+P)^{-1 / 2}=W\left(I+P_{0}^{1 / 2}\right)^{-1}\left(I+P_{0}^{1 / 2}\right)(I+P)^{-1 / 2}, P_{0}=-\Delta$, and it is known that the product of the first two factors is compact and the product of the second two factors is bounded. So, $W(I+P)^{-1 / 2}$ is compact. Now if we multiply by the bounded operator $(I+P)^{-1 / 2}$ on the right, we conclude that $W(I+P)^{-1}$ is compact.

The following theorems show that under certain conditions on $V$, the essential spectrum of $P$ is empty, so the spectrum is purely discrete.

3.3. Theorem: Let $V: \mathbb{R}^{n} \rightarrow \mathbb{R}$ such that $V(x) \rightarrow \infty$ for $|x| \rightarrow \infty$, then $\sigma_{e s s}(-\Delta+V)$ is empty. Proof: For any $E \in \mathbb{R}$, write $V-E=f-g$ where $f \geq 0$ and $g$ has compact support. By the Lemma 3.2, $g$ is $(-\Delta+f)$-compact, so by virtue of perturbation theorem of Weyl we have $\sigma_{e s s}(-\Delta+f)=\sigma_{e s s}(P-E)$.

Since $f \geq 0$, we know that $\sigma_{e s s}(P-E) \subset\left[0,+\infty\left[\right.\right.$ and then $\sigma_{e s s}(P) \subset[E,+\infty[$. Since this is true for all $E$, we conclude that $\sigma_{e s s}(P)$ is empty. The next result is due to Friedrichs (1934).

3.4. Theorem: Let $V: \mathbb{R}^{n} \rightarrow \mathbb{R}$ be continuous with $V(x) \rightarrow \infty$ for $|x| \rightarrow \infty$. Then:

1) There is a constant $C_{0}$ such that $P+C_{0} \geq 1$ and $\left(P+C_{0}\right)^{-1}$ is compact.

2) The spectrum $\sigma(P)$ of $P$ is an increasing sequence $\left(\lambda_{k}\right)_{k \in \mathbb{N}} \subset \mathbb{R}$ of eigenvalues of finite multiplicity and $\lambda_{k} \rightarrow \infty$ for $k \rightarrow \infty$. In particular, $\sigma(P)=\sigma_{\text {disc }}(P)$ and $\sigma_{e s s}(P)=\emptyset$.

The associated eigenfunctions form an orthonormal basis of the Hilbert space $L^{2}\left(\mathbb{R}^{n}\right)$.

Proof: 1) Clearly, $V(x) \geq-C$ for some $C$ and therefore $P$ is self-adjoint. Thus, without loss of generality, we assume that $V(x) \geq 0, x \in \mathbb{R}^{n}$, which implies that $P \geq 0$. Let $f_{k}$ be a weakly converging sequence to 0 in $L^{2}\left(\mathbb{R}^{n}\right)$ and let $u_{k}=(P+I)^{-1} f_{k}, k \in \mathbb{N}$.

We show that there is a subsequence $\left(u_{k_{j}}\right)_{j \in \mathbb{N}}$ such that $\left\|u_{k_{j}}\right\| \rightarrow 0$ as $j \rightarrow \infty$. First, the fact that $\left(f_{k}\right)_{k \in \mathbb{N}}$ converges weakly implies that $\left(f_{k}\right)_{k \in \mathbb{N}}$ is bounded in $L^{2}\left(\mathbb{R}^{n}\right)$. As $(P+I)^{-1}$ is bounded, the sequence $\left(u_{k_{j}}\right)_{j \in \mathbb{N}}$ is also bounded in $L^{2}\left(\mathbb{R}^{n}\right)$ and converges weakly to zero in $L^{2}\left(\mathbb{R}^{n}\right)$.

Furthermore, $u_{k} \in D(P)$ and $\left\|P u_{k}\right\| \leq\left\|(P+I)(P+I)^{-1} f_{k}\right\|+\left\|u_{k}\right\|=\left\|f_{k}\right\|+$ $\left\|u_{k}\right\| \leq C_{1}, C_{1}>0$. So, $\left\langle P u_{k}, u_{k}\right\rangle \leq|| P u_{k}\left|\left\|\mid u_{k}\right\| \leq C_{2}, C_{2}>0\right.$. As $V(x) \rightarrow \infty$ for $| x \mid \rightarrow$ $\infty$, given $\varepsilon>0$, we choose $R>0$ such that $V(x) \geq \frac{1}{\varepsilon}$ for $|x| \geq R$. 
Since, for all $\varphi \in D(P):\langle P \varphi, \varphi\rangle=\int_{\mathbb{R}^{n}}(-\Delta \varphi(x)+\varphi(x) V(x)) \overline{\varphi(x)} d x=$ $\int_{\mathbb{R}^{n}}\left(|\nabla \varphi(x)|^{2}+V(x)|\varphi(x)|^{2}\right) d x$, we have $\int_{\mathbb{R}^{n}} V(x)\left|u_{k}(x)\right|^{2} d x \leq C_{2}$ and hence, $\int_{x \geq R}\left|u_{k}(x)\right|^{2} d x \leq \varepsilon C_{2}$.

Let $\psi_{R} \in C_{c}^{\infty}\left(\mathbb{R}^{n}\right)$ with Supp $\psi \subset B_{2 R}, \psi=1$ on $B_{R}$ and $0 \leq \psi \leq 1$. We observe that: $\left\|\psi_{R} u_{k}\right\|^{2}+\left\|\Delta\left(\psi_{R} u_{k}\right)\right\|^{2} \leq 2 \int_{\mathbb{R}^{n}} \psi_{R}^{2}(x)\left|\nabla u_{k}(x)\right|^{2} d x+2 \int_{\mathbb{R}^{n}}\left|\nabla \psi_{R}(x)\right|^{2}\left|u_{k}(x)\right|^{2} d x+2\left\|u_{k} \quad\right\|^{2} \leq C_{3}, C_{3}>0$.

Consequently, $\left(\psi_{R} u_{k}\right)_{k \in \mathbb{N}}$ is a bounded sequence in the graph norm. Applying Rellich's compactness theorem, we obtain a subsequence $\left(u_{k_{j}}\right)_{j \in \mathbb{N}}$ of $\left(u_{k}\right)_{k \in \mathbb{N}}$ such that $\left\|\psi_{R} u_{k j}\right\| \rightarrow 0$ as $u_{k}$ converges weakly to 0 . Choose $j_{0} \in \mathbb{N}$ so that $\left\|\psi_{R} u_{k_{j}}\right\|^{2} \leq \varepsilon$ for every $j \geq j_{0}$. So, $\left\|u_{k_{j}}\right\|^{2} \leq \varepsilon C_{2}+\varepsilon$ for $j \geq j_{0}$.

2) Note that $(P+I): D(P) \rightarrow L^{2}\left(\mathbb{R}^{n}\right)$ is bijective with compact inverse $(P+I)^{-1}$. This implies that $\sigma(A)$ is either empty or $\sigma(P)=\sigma_{\text {disc }}(P)$ contains at most countably many eigenvalues $\lambda_{k}$. If $\sigma(P)$ is infinite, then $\left(\lambda_{k}\right)_{k \in \mathbb{N}}$ is an increasing sequence of eigenvalues of finite multiplicity and $\left|\lambda_{k}\right| \rightarrow \infty$ as $k \rightarrow \infty$. For all $\lambda_{k} \in \sigma(P)$, the range $\mathcal{R}\left(\lambda_{k}-P\right)$ of $\lambda_{k}-P$ is closed, $\operatorname{dim} \mathcal{N}\left(\lambda_{k}-P\right)=\operatorname{codim} \mathcal{R}\left(\lambda_{k}-P\right)$, and thus the eigenfunctions of $(P+I)^{-1}$ form an orthonormal basis of $L^{2}\left(\mathbb{R}^{n}\right)$, where $\mathcal{N}\left(\lambda_{k}-P\right)$ denotes the kernel of $\left(\lambda_{k}-P\right)$. Clearly, $\sigma_{e s s}(P)=\emptyset$.

3.5. Example: Let $V(x)=x^{2}, x \in \mathbb{R}$. Then, $P=-\frac{d^{2}}{d x^{2}}+x^{2}: C_{c}^{\infty}(\mathbb{R}) \rightarrow L^{2}(\mathbb{R})$ is self-adjoint on $H^{2}(\mathbb{R}) \cap\left\{\varphi \in L^{2}(\mathbb{R}): x^{2} \varphi \in L^{2}(\mathbb{R})\right\}$. Theorem 3.4 implies that $\sigma(P)=\sigma_{\text {disc }}(P)$ consists of a sequence of eigenvalues of finite multiplicity $0<\lambda_{1}<\lambda_{2}<\cdots$ with $\lambda_{k} \rightarrow \infty$ as $k \rightarrow \infty$. It is possible to calculate the eigenvalues of $P$ explicitly, they are given by $\lambda_{k}=2 k+1 ; k \in$ $\mathbb{N}$. The associated eigenfunctions are $\phi_{k}(x)=c_{k} Q_{k}(x) e^{-x^{2} / 2}, c_{k} \in \mathbb{R}$, where $Q_{k}(x)$ is the $k$ thorder Hermite polynomial, $k \in \mathbb{N}$.

3.2. $\boldsymbol{V}(\boldsymbol{x}) \rightarrow \mathbf{0}$ for $|\boldsymbol{x}| \rightarrow \infty$. If $V$ is locally bounded and $V(x) \rightarrow 0$ as $|x| \rightarrow \infty$, we show that under certain conditions, the essential spectrum of $P=-\Delta+V: D(P) \rightarrow L^{2}\left(\mathbb{R}^{n}\right)$ is in fact exactly the set of non-negative real numbers. A special treatment is reserved for Schrödinger operators with Coulomb potentials $V(x)=\frac{\gamma}{|x|}, \gamma>0$.

3.6. Theorem: Let $V: \mathbb{R}^{n} \rightarrow \mathbb{R}$ be locally bounded and $V(x) \rightarrow 0$ as $|x| \rightarrow \infty$. Then, $\sigma_{\text {ess }}(P)=$ $[0,+\infty[$. 
Proof: The proof of Theorem 3.4 allows to see that $P$ can have only isolated eigenvalues of finite multiplicity on $]-\infty, 0[$. It remains for us to show that $[0,+\infty[\subset \sigma(P)$. Let $\lambda \geq 0$ be fixed. We shall construct a Weyl sequence $\left(\varphi_{k}\right)_{k \in \mathbb{N}^{*}} \subset H^{2}\left(\mathbb{R}^{n}\right)$ of $P$ and $\lambda$. We have $-\Delta e^{i \xi \cdot x}=$ $\lambda e^{i \xi \cdot x}$, where $\xi \in \mathbb{R}^{n},|\xi|=\sqrt{\lambda}$ and $\lim _{|x| \rightarrow \infty}(P-\lambda) e^{i \xi \cdot x}(P-\lambda) e^{i \xi \cdot x}$ since $\lim _{|x| \rightarrow \infty} V(x)=0$. Moreover, let $\psi \in C_{c}^{\infty}\left(\mathbb{R}^{n}\right)$ such that $\psi \geq 0, \psi(x)=1$ for $|x| \leq \frac{1}{2}$ and $\psi(x)=0$ for $|x| \geq$ 2. Let $\psi_{k}(x)=\psi\left(\frac{x-k}{\sqrt{k}}\right), k \in \mathbb{N}^{*}$, then:

$\operatorname{Supp} \psi_{k} \subset\{x \in \mathbb{N}:|x-k| \leq \sqrt{k}\}$ and $\lim _{k \rightarrow \infty} \sup _{x \in S u p p} \psi_{k} V(x)=0$.

Now consider the sequence $\varphi_{k}(x)=\psi_{k}(x) e^{i \xi \cdot x}, k \in \mathbb{N}^{*}$. Note that for every $k \in \mathbb{N}^{*}$ : $\left\|\varphi_{k}\right\|^{2}=\int_{\mathbb{R}^{n}}\left|\psi_{k}(x)\right|^{2} d x=k^{n / 2}\|\psi\|^{2}$.

So, $P \varphi_{k}=\left(-\Delta \psi_{k}\right) e^{i \xi \cdot x}-\left(\nabla \psi_{k}\right)\left(-\nabla e^{i \xi \cdot x}\right)+|\xi|^{2} \psi_{k}(x) e^{i \xi \cdot x}+V(x) \psi_{k}(x) e^{i \xi \cdot x}$ and $(P-\lambda) \varphi_{k}=e^{i \xi \cdot x}\left(P \psi_{k}-i \xi \cdot \nabla \psi_{k}\right)$.

Now, $\left|\nabla \psi_{k}\right| \leq \frac{\|\psi\|^{2}}{\sqrt{k}}$ and $\left|\Delta \psi_{k}\right| \leq \frac{\|\psi\|^{2}}{k}, k \in \mathbb{N}^{*}$. We can deduce from (3.3), that $\lim _{k \rightarrow \infty} \sup _{x \in \mathbb{R}^{n}}\left|(P-\lambda) \varphi_{k}(x)\right|=0$, and thus $\lim _{k \rightarrow \infty} \frac{\left\|(P-\lambda) \varphi_{k}\right\|^{2}}{k^{n / 2}}=0$. Along with (3.2) this implies $\lim _{k \rightarrow \infty} \frac{\left\|(P-\lambda) \varphi_{k}\right\|^{2}}{\left\|\varphi_{k}\right\|}=0$.

Sketch of the proof of Theorem 3.6, or directly thanks to Weyl's theorem, we can prove the theorem for Schrödinger operators with relatively bounded potentials with respect to $-\Delta$ and with relative bound $<1$.

3.7. Theorem: Let $V: \mathbb{R}^{n} \rightarrow \mathbb{R}$ be piecewise continuous with $V(x) \rightarrow 0$ as $|x| \rightarrow \infty$. Assume that the multiplication operator by $V$ is relatively bounded with respect to $-\Delta$ with relative bound $<1$. Then $P=-\Delta+V: D(P)=H^{2}\left(\mathbb{R}^{n}\right) \rightarrow L^{2}\left(\mathbb{R}^{n}\right)$ is self-adjoint and $\sigma_{e s s}(P)=$ $\sigma_{\text {ess }}(-\Delta)=[0,+\infty[$.

3.8. Remark: Under the assumptions of Theorem 3.7, we have that $\sigma_{e s s}(P)=[0,+\infty[$. Nevertheless, it is possible that $P$ has discrete eigenvalues below 0 (and they are of importance in physics when thinking of spectroscopy etc.). These eigenvalues are characterized by the minmax-principle.

3.9. Theorem: Let $P$ as in Theorem 3.7. If there exists $\varphi \in H^{2}\left(\mathbb{R}^{n}\right)$ with $\langle P \varphi, \varphi\rangle=$ $\int_{\mathbb{R}^{n}} P \varphi(x) \overline{\varphi(x)} d x<0$, then $P$ has at least one negative eigenvalue.

Proof: If we suppose that $\sigma(P) \subset[0,+\infty[$, as $P$ is self-adjoint we would deduce that $P$ is positive, i.e. $\langle P u, u\rangle \geq 0$ for all $u \in H^{2}\left(\mathbb{R}^{n}\right)$, what contradicts the assumption $\langle P \varphi, \varphi\rangle<0$. 
3.10. Example: We focus in particular on the Coulomb potential $V(x)=-\frac{1}{|x|}, x \in \mathbb{R}^{3} \backslash$ \{0\}. $P=-\Delta-\frac{1}{|x|}$ in $L^{2}\left(\mathbb{R}^{3}\right)$ is the Schrödinger operator of the hydrogen atom. Hardy's inequality implies that the Coulomb potential in $\mathbb{R}^{3}$ is relatively bounded with respect to $-\Delta$ with relative bound 0 and the perturbation theorem of Kato and Rellich shows that $P$ is selfadjoint on $H^{2}\left(\mathbb{R}^{3}\right)$, we have also $\sigma_{e s s}(P)=\sigma_{e s s}(-\Delta)=[0,+\infty[$.

Indeed, first check that $V \psi \in L^{2}\left(\mathbb{R}^{3}\right)$ for all $\psi \in H^{2}\left(\mathbb{R}^{3}\right)$. Let $\varphi=\mathcal{F}^{-1} \psi \in L^{2}\left(\mathbb{R}^{3}\right)$, so $\psi=$ $\mathcal{F} \varphi$ where $\mathcal{F}$ and $\mathcal{F}^{-1}$ are the Fourier transform and inverse Fourier transform, respectively on $L^{2}\left(\mathbb{R}^{3}\right)$. As the functions of $H^{2}\left(\mathbb{R}^{3}\right)$ are continuous and tend to zero at infinity, we deduce that $\psi$ is essentially-bounded on $\mathbb{R}^{3}$ :

$$
\begin{aligned}
& \|\psi\|_{\infty}=\operatorname{supess}_{x \in \mathbb{R}^{3}}|\psi(x)|=\sup _{x \in \mathbb{R}^{3}}\left|(2 \pi)^{-3 / 2} \int_{\mathbb{R}^{3}} e^{-i x \cdot \xi} \varphi(\xi) d \xi\right| \\
& \leq(2 \pi)^{-3 / 2} \int_{|\xi| \leq 1}|\varphi(\xi)| d \xi+(2 \pi)^{-3 / 2} \int_{|\xi|>1}|\xi|^{-2}\left(|\xi|^{2}|\varphi(\xi)|\right) d \xi .
\end{aligned}
$$

Hence, we can apply the Cauchy-Schwarz inequality and obtain:

$\|\psi\|_{L^{\infty}\left(\mathbb{R}^{3}\right)} \leq c_{1}\|\psi\|_{L^{2}\left(\mathbb{R}^{3}\right)}+c_{2}\left\||\xi|^{2} \varphi\right\|_{L^{2}\left(\mathbb{R}^{3}\right)}$

$c_{1}=(2 \pi)^{-3 / 2}\left(\left(\frac{4 \pi}{3}\right)^{1 / 2}\right.$ and $c_{2}=(2 \pi)^{-3 / 2}\left(\int_{|\xi>1|} \frac{d \xi}{|\xi|^{4}}\right)^{1 / 2}$

$\left(\psi \in H^{2}\left(\mathbb{R}^{3}\right)\right)$, then $\Delta \psi \in L^{2}\left(\mathbb{R}^{3}\right)$, and $|\xi|^{2} \varphi=\mathcal{F}^{-1}(-\Delta \psi) \in L^{2}\left(\mathbb{R}^{3}\right)$.

Let us also use the fact that the Fourier transform is a unitary operator on $L^{2}\left(\mathbb{R}^{3}\right)$, and obtain $\|\psi\|_{L^{\infty}\left(\mathbb{R}^{3}\right)} \leq c_{1}\|\psi\|_{L^{2}\left(\mathbb{R}^{3}\right)}+c_{2}\|\Delta \psi\|_{L^{2}\left(\mathbb{R}^{3}\right)}$.

So, for all $\varepsilon>0$ :

$\|V \psi\|_{L^{2}\left(\mathbb{R}^{3}\right)}^{2}=\int_{r=|x| \leq \varepsilon} r^{-2}|\psi(x)|^{2} d x+\int_{r>\varepsilon} r^{-2}|\psi(x)|^{2} d x$

or,

$\|V \psi\|_{L^{2}\left(\mathbb{R}^{3}\right)} \leq\|\psi\|_{L^{\infty}\left(\mathbb{R}^{3}\right)} \sqrt{\int_{r \leq \varepsilon} r^{-2} d x}+\varepsilon^{-1}\|\psi\|_{L^{2}\left(\mathbb{R}^{3}\right)}$.

Using (3.4) we also have: $\|V \psi\|_{L^{2}\left(\mathbb{R}^{3}\right)} \leq a\|\Delta \psi\|_{L^{2}\left(\mathbb{R}^{3}\right)}+b\|\psi\|_{L^{2}\left(\mathbb{R}^{3}\right)}$

with $a=c_{2} \sqrt{\int_{r \leq \varepsilon} r^{-2} d x}$ and $b=\varepsilon^{-1}+c_{1} \sqrt{\int_{r \leq \varepsilon} r^{-2} d x}$. Thus, $V$ is $\Delta$-bounded with relative bound $a$, make $a$ small enough by choosing $\varepsilon \rightarrow 0^{+}$. 
For the Coulomb potential in $\mathbb{R}^{3}$ one obtains an infinite sequence of negative eigenvalues. As $V(x)=-\frac{1}{x}$ is spherically symmetric, $V(x)=V(r)$, with $r=|x|$, the main idea is to separate $P=-\Delta-\frac{1}{r}$ in spherical coordinates to obtain the negative eigenvalues and the associated eigenfunctions. For any $x \in \mathbb{R}^{3} \backslash\{0\}, x=r \omega$ where $\omega=\frac{x}{|x|} \in S^{2}$ the unit sphere of $\mathbb{R}^{3}$.

The operator $-\Delta$ on $S^{2}$, has compact resolvent and purely discrete spectrum: $0=v_{0}<$ $v_{1}<\cdots<v_{k} \rightarrow \infty$ as $k \rightarrow \infty$, the associated eigenspaces to $v_{k}$ have a basis of $C^{\infty}$-functions $\psi_{k, l}: S^{2} \rightarrow \mathbb{R}, l=1, \ldots, m_{k}$, called the spherical harmonics, where $m_{k}=k(k+1), k \in \mathbb{N}^{*}$, is the dimension of the eigenspace.

Using separation of variables $u(x)=f(r) \psi_{k, l}(\omega)$ for the eigenfunctions $u$ and eigenvalues $\lambda$ of $P=-\Delta-\frac{1}{|x|}$ in the Hilbert space $L^{2}\left(\mathbb{R}^{3}\right)=L^{2}(] 0,+\infty\left[, r^{2} d r\right) \oplus$ $L^{2}\left(S^{2}, d \sigma_{2}\right)$, leads to the Bessel differential equation for $f$ :

$$
\left.-f^{\prime \prime}(r)-\frac{2}{r} f^{\prime}(r)-\frac{1}{|x|} f(r)+\frac{v_{k}}{r^{2}} f(r)=\lambda f(r), r \in\right] 0,+\infty[.
$$

We remark that one can show by power-series methods that for any $k \in \mathbb{N}^{*}$, there exists a solution $f=f_{k} \in L^{2}(] 0,+\infty\left[, r^{2} d r\right)$ and an infinite sequence $\lambda_{k}$ of negative eigenvalues of $P, \lambda_{k}=-\frac{1}{4 k^{2}}, k \in \mathbb{N}^{*}$ (see Landau and Lifshitz, 1977).

So, we see that the hydrogen atom has an infinite number of bound states below the essential spectrum $\left(\sigma_{e s s}(P)=[0,+\infty[)\right.$, which accumulate at zero.

\subsection{Bounded Continuous Potentials}

If $V: \mathbb{R}^{n} \rightarrow \mathbb{R}$ is bounded and piecewise continuous, then $P=-\Delta+V$ is self-adjoint with domain $H^{2}\left(\mathbb{R}^{n}\right)$. Let $s_{-}=\inf _{x \in \mathbb{R}^{n}} V(x)$ and $s_{+}=\sup _{x \in \mathbb{R}^{n}} V(x)$. It's clear that $\inf \sigma(P) \in\left[s_{-}, s_{+}\right]$and $\sigma(P) \subset\left[s_{-},+\infty\left[\right.\right.$. The following result gives a location of the spectrum of $P$ around $\left[s_{-}, s_{+}\right]$.

3.11. Theorem: Let $V: \mathbb{R}^{n} \rightarrow \mathbb{R}$ be bounded and piecewise continuous and let $\lambda \geq 0$. Then $\sigma(P) \cap\left[\lambda-r_{+}, \lambda+r_{+}\right] \neq \emptyset$, where $r_{+}=\max \left(\left|s_{-}\right|,\left|s_{+}\right|\right)$.

Proof: Since, $\sigma_{e s s}(-\Delta)=\left[0,+\infty\left[\right.\right.$, there exists a singular sequence $\left(\varphi_{k}\right)_{k \in \mathbb{N}^{*}}$ in $H^{2}\left(\mathbb{R}^{n}\right)$ associated to $-\Delta$ and $\lambda$. Since $P$ is self-adjoint on $H^{2}\left(\mathbb{R}^{n}\right)$, let $\left(E_{\mu}\right)_{\mu \in \mathbb{R}}$ be the spectral family associated with $P$. The spectral theorem yields:

$$
\inf _{\mu \in \sigma(P)}|\mu-\lambda|^{2}\left\|\varphi_{k}\right\|^{2} \leq \int_{\mathbb{R}}(\mu-\lambda)^{2} d\left\langle E_{\mu} \varphi_{k}, \varphi_{k}\right\rangle=\left\|(P-\lambda) \varphi_{k}\right\|^{2} \leq\left(\left\|(-\Delta-\lambda) \varphi_{k}\right\|+\left\|V \varphi_{k}\right\|\right)^{2}
$$


As $\left\|\varphi_{k}\right\|=1,\left\|V \varphi_{k}\right\| \leq r_{+}$for all $k \in \mathbb{N}$ and $\left\|(-\Delta-\lambda) \varphi_{k}\right\| \rightarrow 0$ as $k \rightarrow \infty$, we obtain that: $\inf _{\mu \in \sigma(P)}|\mu-\lambda|^{2} \leq r_{+}^{2}$, thus the gap between $\lambda$ and $\sigma(P)$ is less than $r_{+}$.

3.12. Remark: Simon (2009) showed that $P=-\Delta+V$ has purely discrete spectrum if $V \geq 0$ and, for all $M$, the set $\left\{x \in \mathbb{R}^{n}: V(x)>M\right\}$ has finite Lebesgue measure. We may now prove a beneficial result, namely that under "small" variations of $V$, the essential spectrum remains the same.

3.13. Theorem: Let $V_{1}$ and $V_{2}$ be two bounded potentials on $\mathbb{R}^{n}$ satisfying:

$\lim _{|x| \rightarrow \infty}\left|V_{1}(x)-V_{2}(x)\right|=0$. Then, $\sigma_{e s s}\left(-\Delta+V_{1}\right)=\sigma_{e s s}\left(-\Delta+V_{2}\right)$.

Proof: Let $P_{j}=-\Delta+V_{j}, j=1,2$, on $L^{2}\left(\mathbb{R}^{n}\right)$ with respective domain $H^{2}\left(\mathbb{R}^{n}\right)$. Note that $P_{2}=$ $P_{1}+W$ where, $W=V_{2}-V_{1}$ is bounded and vanishes at infinity. If $\lambda \notin \sigma\left(P_{1}\right) \cup \sigma\left(P_{2}\right)$, then $W\left(P_{1}-z\right)^{-1}=W(-\Delta-z)^{-1}(-\Delta-z)\left(P_{1}-z\right)^{-1}$ or $(-\Delta-z)\left(P_{1}-z\right)^{-1}$ is bounded from $L^{2}\left(\mathbb{R}^{n}\right)$ to $H^{2}\left(\mathbb{R}^{n}\right) . W(-\Delta-z)^{-1}$ is compact by Theorems 3.6 and 3.7. We deduce that $P_{1}$ and $P_{2}$ have the same essential spectrum by perturbation theorem of Weyl.

3.14. Example: Let $P_{\alpha}, 0<\alpha<1$, be the operator with domain $H^{2}(\mathbb{R})$ in $L^{2}(\mathbb{R})$ defined by: $P_{\alpha} \varphi=-\frac{d^{2}}{d x^{2}}+\sin |x|^{\alpha} \varphi(x)$. Clearly, $V(x)=\sin |x|^{\alpha}$ is bounded on $\mathbb{R}$ and $\sigma\left(P_{\alpha}\right) \subset[-1,+\infty[$ since $-1 \leq V(x) \leq 1$.

Firstly, we show that $[-1,1] \subset \sigma_{e s s}\left(P_{\alpha}\right)$. Since $V$ is even and $V^{\prime}(x)=\alpha x^{\alpha-1} \cos x^{\alpha} \rightarrow$ 0 , as $x \rightarrow \infty$, finite-increments formula ensures that for $\lambda \in[-1,1]$ and $\varepsilon_{k}=\frac{1}{k}, k \in \mathbb{N}^{*}$, there exists $x_{k}>0$ so that $|V(x)-\lambda|<\varepsilon_{k}$ for all $x \in B_{2 k}\left(x_{k}\right) .\left(x_{k}\right)_{k \in \mathbb{N}^{*}}$ is monotonically increasing sequence and $x_{k} \rightarrow \infty$. We can also assume that $B_{2 k}\left(x_{k}\right) \cap B_{2(k+1)}\left(x_{k+1}\right)=\emptyset$. Let $\psi_{k} \in C_{c}^{\infty}(\mathbb{R})$ be a cut-off function with $0 \leq \psi_{k} \leq 1, \psi_{k}=1$ on $]-k, k\left[\right.$ and $\operatorname{Supp} \psi_{k} \subset$ ] $2 k, 2 k\left[\right.$. Let $\varphi_{k}=\frac{\psi_{k}\left(x-x_{k}\right)}{\left\|\psi_{k}\left(x-x_{k}\right)\right\|}$. Clearly, $\varphi_{k} \rightarrow 0$ weakly and $\left\|\left(P_{\alpha}-\lambda\right) \varphi_{k}\right\| \leq\left\|-\frac{d^{2} \varphi_{k}}{d x^{2}}\right\|+$ $\left\|(V-\lambda) \varphi_{k}\right\| \leq\left\|-\frac{d^{2} \varphi_{k}}{d x^{2}}\right\|+\varepsilon_{k} \rightarrow 0, k \rightarrow \infty$, we see that $\lambda \in \sigma\left(P_{\alpha}\right)$.

Secondly, we show that $\left[1,+\infty\left[\subset \sigma\left(P_{\alpha}\right)\right.\right.$. Let $\lambda \in\left[1,+\infty\left[\right.\right.$, again we choose points $x_{k}$ on the positive half-axis such that $|V(x)|<\varepsilon_{k}$ for all $x \in B_{2 k}\left(x_{k}\right)$. Let $u_{k}, k \in \mathbb{N}^{*}$, be the singular sequence of $\left(-\frac{d^{2}}{d x^{2}}\right)$ and $\lambda$ obtained in the proof of Proposition 2.1. Then, $v_{k}(x)=$ $u_{k}\left(x-x_{k}\right)$ satisfies $\left\|u_{k}\right\|=1$ for all $k \in \mathbb{N}^{*}$, and $v_{k} \rightarrow 0$ weakly as $k \rightarrow \infty$,

$\left\|\left(P_{\alpha}-\lambda\right) v_{k}\right\| \leq\left\|\left(-\frac{d^{2}}{d x^{2}}-\lambda\right) v_{k}\right\|+\left\|V v_{k}\right\| \leq\left\|\left(-\frac{d^{2}}{d x^{2}}-\lambda\right) v_{k}\right\|+\varepsilon_{k} \rightarrow 0, k \rightarrow \infty$, thus $\lambda \in$ $\sigma_{e s s}\left(P_{\alpha}\right)$. Finally, $\sigma\left(P_{\alpha}\right)=\sigma_{e s s}\left(P_{\alpha}\right)=[-1,+\infty[$. 


\subsection{Periodic Potentials}

The case where $V$ does not have a limit as $|x| \rightarrow \infty$ in any direction one might expect the analysis of periodic Schrödinger operators to be difficult. Consider a particle moving in a periodic potential. A primary example of this situation is an electron moving in the potential created by ions or atoms of a solid crystal lattice. Linear operators with periodic coefficients arise in many problems both of classical mechanics, mathematics and modern mathematical physic. Such operators appear, e.g. in solid state physics. More details can be found in Cycon et al. (1987). The possible energies of a free electron, corresponding to the free Schrödinger operator $-\Delta$ form a straightforward continuum $[0,+\infty[$. The introduction of the periodic perturbation $V$ "opens up" gaps in this spectrum. This is called the band structure. In fact the operator $P=-\Delta+V$ can be broken into a family of operators $P_{\theta}$ on a n-torus, each $P_{\theta}$ having discrete spectrum. More precisely, the spectrum of $P_{\theta}$ consists of an increasing sequence of eigenvalues $\lambda_{1}(k) \leq \lambda_{2}(k) \leq \cdots$ tending to infinity. The manner in which the operators $P_{\theta}$ are obtained is not quite unique. Here, we only skim some results for periodic Schrödinger operators. A classical example is the Mathieu operator $-\frac{d^{2}}{d x^{2}}+\mu \cos x$ in $L^{2}\left(\mathbb{R}^{n}\right), \mu \neq 0$.

In $L^{2}\left(\mathbb{R}^{3}\right)$ we study the Schrödinger operator $P=-\Delta+V$ where the potential $V$ is a periodic, bounded, real-valued function on $\mathbb{R}^{3}$. The periodicity of $V$ is defined as follows.

Let $\left\{\gamma_{1}, \gamma_{2}, \gamma_{3}\right\}$ be a set of 3 linearly independent vectors in $\mathbb{R}^{3}$. Construct a crystal by fixing identical particles at the points of the lattice:

$$
\Gamma=\left\{p_{1} \gamma_{1}+p_{2} \gamma_{2}+p_{3} \gamma_{3}: p_{1}, p_{2}, p_{3} \in \mathbb{Z}\right\} \text {. }
$$

For example, if the $\gamma_{j}$ 's are the standard basis for $\mathbb{R}^{3}$, then $\Gamma=\mathbb{Z}^{3} . V$ is said periodic with respect to $\Gamma$ if $V(x+\gamma)=V(x)$, for all $x \in \mathbb{R}^{3}$ and $\gamma \in \Gamma$. $\Gamma$ is called the period lattice of the potential $V$. In that case, $P$ is self-adjoint and lower semibounded on its natural domain, the Sobolev space $H^{2}\left(\mathbb{R}^{3}\right)$. $P$ commutes with the lattice translations, $T_{\gamma} P=P T_{\gamma}$, for all $\gamma \in \Gamma$, where $T_{\gamma}: L^{2}\left(\mathbb{R}^{3}\right) \rightarrow L^{2}\left(\mathbb{R}^{3}\right)$ and $\left(T_{\gamma} \varphi\right)(x)=\varphi(x+\gamma) . T_{\gamma}$ are unitary operators on $L^{2}\left(\mathbb{R}^{3}\right)$ satisfying $T_{\gamma}\left(H^{2}\left(\mathbb{R}^{3}\right)\right) \subset H^{2}\left(\mathbb{R}^{3}\right), T_{\gamma} T_{\mu}=T_{\gamma+\mu}, \gamma, \mu \in \Gamma$.

This will permit us to decompose $P$ as an hilbertian integral. More precisely, we first define $\Omega$ to be the basic lattice cell of the lattice $\Gamma$, i.e. the cell spanned by the basis vectors $\gamma_{1}, \gamma_{2}, \gamma_{3}$ of $\mathbb{R}^{3}$. Let $\Gamma^{*}$ be the lattice dual to $\Gamma$, i.e. the lattice with basis $\gamma_{1}^{*}, \gamma_{2}^{*}, \gamma_{3}^{*}$ satisfying $\gamma_{j}^{*}\left(\gamma_{l}\right)=\delta_{j l}$, the Kronecker symbol, and let $\Omega^{*}$ be a basic lattice cell of $\Gamma^{*}$. Now for each $\theta \in$ $\Omega^{*}$, we define the Hilbert space $H_{\theta}=L^{2}(\Omega)$ and we then define the Hilbert space $H$ to be the direct fiber integral, $H=\int_{\Omega^{*}}^{\oplus} H_{\theta} d \theta$ where $d \theta$ is the standard Lebesgue measure on $\Omega^{*}$ 
normalized so that $\int_{\Omega^{*}}^{\oplus} d \theta=1$. In fact, $H=L^{2}\left(\Omega^{*}, d \theta, L^{2}(\Omega)\right)$ is the Hilbert space of square integrable $L^{2}(\Omega)$-valued functions and if $\psi \in H, \psi=\left(\psi_{\theta}\right)_{\theta \in \Omega^{*}}$.

Let $P_{\theta}$ be the restriction of the operator $P$ to $H_{\theta}$ with domain consisting of functions $\psi_{\theta} \in H_{\theta} \cap H^{2}\left(\mathbb{R}^{3}\right)$ satisfying the boundary conditions $T_{\gamma} \psi(x)=e^{i \theta \gamma} \psi(x)$ for the basis elements $\gamma=\gamma_{1}, \gamma_{2}, \gamma_{3}$. Define now the operator $\psi_{\theta}(x) \mapsto P_{\theta} \psi_{\theta}(x)$ on $H=\int_{\Omega^{*}}^{\oplus} H_{\theta} d \theta$ and denote it $H=\int_{\Omega^{*}}^{\oplus} P_{\theta} d \theta$. Let us consider the Floquet transform $[F \varphi]_{\theta}(x)=\sum_{\gamma \in \Gamma} e^{-i \theta \gamma} T_{\gamma} \varphi(x)$ from $L^{2}\left(\mathbb{R}^{3}\right)$ into $H$. The properties of such operators are summarized in the following.

3.15. Theorem: 1) $P_{\theta}$ and $\int_{\Omega^{*}}^{\oplus} P_{\theta} d \theta$ are self-adjoint on $H_{\theta}$ and $H$ respectively, for all $\theta \in \Omega^{*}$.

2) The operator $F$ extends uniquely to a unitary operator and $F P F^{-1}=\int_{\Omega^{*}}^{\oplus} P_{\theta} d \theta$.

3) The spectrum of $P_{\theta}$ is purely discrete for all $\theta \in \Omega^{*}$ and $\sigma(P)=\bigcup_{\theta \in \Omega^{*}} \sigma\left(P_{\theta}\right)$.

Proof: 1) is automatic by construction.

2) $F$ is an isometry on $C_{c}^{\infty}\left(\mathbb{R}^{3}\right)$. Indeed, using Fubini's theorem one has:

$$
\begin{gathered}
\|F \psi\|_{H}^{2}=\int_{\Omega^{*}}\left\|F(\psi)_{\theta}\right\|_{H}^{2} d \theta=\int_{\Omega^{*}} \int_{\Omega}\left|\sum_{\gamma \in \Gamma} e^{-i \theta \gamma} T_{\gamma} \psi(x) d x\right|^{2} d \theta \\
=\int_{\Omega}\left(\sum_{\gamma, \alpha \in \Gamma} T_{\gamma} \psi(x) \overline{T_{\alpha} \varphi(x)} \int_{\Omega^{*}} e^{-i \theta \gamma} e^{-i \theta \alpha}\right) d x \\
=\int_{\Omega} \sum_{\gamma \in \Gamma}\left|T_{\gamma} \psi(x)\right|^{2} d x=\int_{\mathbb{R}^{3}}|\psi(x)|^{2} d x=\|\psi\|_{L^{2}\left(\mathbb{R}^{3}\right)^{.}}^{2}
\end{gathered}
$$

Hence, $\|F \psi\|_{H}=\|\psi\|_{L^{2}\left(\mathbb{R}^{3}\right)}$ and by density $F$ extends to an isometry on all of $H$. To show that $F$ is in fact a unitary operator, we naturally define $F^{*}: H \rightarrow H$ by the formula:

$$
F^{*} g(x+\gamma)=\int_{\Omega^{*}} e^{i \theta \gamma} g_{\theta}(x) d \theta, \gamma \in \Gamma \text { and } x \in \Omega .
$$

It's clear that a direct computation shows that $F^{*}$ is the adjoint of $F$ and is also an isometry, so $F$ is a unitary operator and $F P F^{*}=\int_{\Omega^{*}}^{\oplus} P_{\theta} d \theta$.

3) The resolvents of $P$ and $P_{\theta}$ are related by $F(P-z)^{-1} F^{-1}=\int_{\Omega^{*}}^{\oplus}\left(P_{\theta}-z\right)^{-1} d \theta$.

Then, $(P-z)$ is boundedly invertible if and only if each operator $\left(P_{\theta}-z\right), \theta \in \Omega^{*}$, is too, which means that $\sigma(P)=\bigcup_{\theta \in \Omega^{*}} \sigma\left(P_{\theta}\right)$. On the other hand, we know that the spectra of Schrödinger operators on bounded domains with Dirichlet boundary conditions is purely discrete accumulating at $+\infty$ (Cycon et al., 1987). 
This is the case of operators $P_{\theta}, \theta \in \Omega^{*}$, so the spectrum of each $P_{\theta}, \theta \in \Omega^{*}$, is discrete composed from a sequence of eigenvalues with finite multiplicity $\lambda_{0}(\theta) \leq \lambda_{1}(\theta) \leq \cdots \leq$ $\lambda_{l}(\theta) \leq \cdots$ and $\lim _{l \rightarrow+\infty} \lambda_{l}(\theta)=+\infty$. This shows that the spectrum of $P$ is union of the sets $\left\{\lambda_{l}(\theta): \theta \in \Omega^{*}\right\}$. In fact, $\sigma(P)=\bigcup_{l \in \mathbb{N}}\left[\inf _{\theta \in \Omega^{*}} \lambda_{l}(\theta), \sup _{\theta \in \Omega^{*}} \lambda_{l}(\theta)\right]$ this is the so-called band structure of spectrum in solid state physics.

3.16. Remark: One can prove similar results in one dimensional case (Gherib and Messirdi, 2005).

3.17. Example: A classical example is the Mathieu operator $-\frac{d^{2}}{d x^{2}}+\mu \cos x$ in $L^{2}(\mathbb{R}), \mu \neq 0$. $V(x)=\mu \cos x$ is $2 \pi$-periodic continuous function on $\mathbb{R}$. Let's take the previous constructions for $V$ on $\mathbb{R}$, using the approach of Gherib and Messirdi (2005). Let $\left.H^{\prime}=L^{2}\right] 0,2 \pi[$ and $H=$ $\int_{[0,2 \pi}^{\oplus}\left[H^{\prime} \frac{d \theta}{2 \pi}\right.$. Consider the operator $F: L^{2}(\mathbb{R}) \rightarrow H$ defined by:

$$
(F \psi)_{\theta}(x)=\sum_{k \in \mathbb{Z}} e^{-i \theta k} \psi(x+2 k \pi), \theta \in[0,2 \pi[.
$$

$F$ is well-defined for $\psi \in C_{c}^{\infty}(\mathbb{R})$ since the sum is convergent, and has a unique extension to a unitary operator on $L^{2}(\mathbb{R})$. Denote by $P_{0}=-\frac{d^{2}}{d x^{2}}$ the self-adjoint operator in $L^{2}(\mathbb{R})$ with domain $H^{2}(\mathbb{R})$. For $\theta \in\left[0,2 \pi\right.$ [, we consider the self-adjoint operator $P_{0, \theta}=P_{0}$ in $L^{2}[0,2 \pi[$ with the boundary conditions $\psi(2 \pi)=e^{i \theta} \psi(0)$ and $\psi^{\prime}(2 \pi)=e^{i \theta} \psi^{\prime}(0)$. We have:

$F P_{0} F^{-1}=\int_{[0,2 \pi}^{\oplus}\left[P_{0, \theta} \frac{d \theta}{2 \pi}\right.$.

Since, if $\psi \in C_{c}^{\infty}(\mathbb{R})$, then $F \psi \in C^{\infty}$ on $] 0,2 \pi\left[\right.$ with $(F \psi)_{\theta}^{\prime}=\left(F \psi^{\prime}\right)_{\theta}(x)$ (similarly for higher derivatives), moreover $(F \psi)_{\theta}(2 \pi)=e^{i \theta}(F \psi)_{\theta}(0),(F \psi)_{\theta}^{\prime}(2 \pi)=e^{i \theta}(F \psi)_{\theta}^{\prime}(0)$ and $P_{0, \theta}(F \psi)=F\left(-\psi^{\prime \prime}\right)_{\theta}$, for all $\theta \in[0,2 \pi[$.

Now let $P_{\theta}=P_{0, \theta}+V(x)$ as an operator in $L^{2}\left[0,2 \pi\left[\right.\right.$. Then, $F P F^{-1}=\int_{[0,2 \pi}^{\oplus}\left[P_{\theta} \frac{d \theta}{2 \pi}\right.$. Indeed, in view of (3.6), it is enough to show that $F V F^{-1}=F(\mu \cos x) F^{-1}=\int_{[0,2 \pi}^{\oplus}\left[V_{\theta} \frac{d \theta}{2 \pi}\right.$, where $\left(V_{\theta} \psi\right)(x)=\mu \cos x \psi(x)$ on $L^{2}[0,2 \pi[$.

One has for all $\theta \in[0,2 \pi[$,

$$
(F V \psi)_{\theta}(x)=\sum_{k \in Z} e^{-i \theta k} V(x+2 \pi k) \psi(x+2 \pi k)=V(x) \sum_{k \in Z} e^{-i \theta k} \psi(x+2 \pi k)=V_{\theta}(F \psi)_{\theta}(x)
$$

and thus, $F P F^{-1}=F P_{0} F^{-1}+F V F^{-1}=\int_{[0,2 \pi}^{\oplus}\left[\left(P_{0, \theta}+V_{\theta}\right) \frac{d \theta}{2 \pi}=\int_{[0,2 \pi}^{\oplus}\left[P_{\theta} \frac{d \theta}{2 \pi}\right.\right.$. 
Remark that the operator $P_{0, \theta}$ is positive, so $P_{0, \theta}+I$ is boundedly invertible. The inverse $K_{\theta}=\left(P_{0, \theta}+I\right)^{-1}$ is easily and explicitly determined as:

1. $\left(K_{\theta} f\right)(x)=\int_{0}^{2 \pi} G_{\theta}(x, y) f(y) d y$,

$$
G_{\theta}(x, y)=\frac{e^{-|x-y|}}{2}+\frac{e^{x-y}}{2\left(e^{2 \pi-i \theta}-1\right)}+\frac{e^{y-x}}{2\left(e^{2 \pi+i \theta}-1\right)} .
$$

$K_{\theta}$ is Hilbert-Schmidt and so compact operator anlytically dependent on $\theta$ in a neighborhood of $[0,2 \pi]$. Thus, $P_{\theta}$ depends analytically on $\theta$ and has a compact resolvent.

Hence, the spectrum of $P_{\theta}$ is discrete and consists of eigenvalues $\lambda_{0}(\theta) \leq \lambda_{1}(\theta) \leq \cdots \leq$ $\lambda_{l}(\theta) \leq \cdots$ and $\sigma(P)$ is a union of intervals $\left[\inf _{\theta \in[0,2 \pi[} \lambda_{l}(\theta), \sup _{\theta \in[0,2 \pi[} \lambda_{l}(\theta),\right]$. Moreover, $\lambda_{l}(\theta)=$ $\lambda_{l}(2 \pi-\theta), \lambda_{l}(\theta)$ are nondegenerate, hence, depend analytically on $\left.\theta \in\right] 0, \pi[$ and for $l$ odd (respectively, even) $\lambda_{l}(\theta)$ is strictly monotone increasing (respectively, decreasing) on $] 0, \pi[$. Therefore, $\lambda_{0}(\pi)<\lambda_{0}(0) \leq \lambda_{1}(0)<\lambda_{1}(\pi) \leq \cdots \leq \lambda_{2 l-1}(0)<\lambda_{2 l-1}(\pi) \leq \lambda_{2 l}(\pi)<\lambda_{2 l}(0)$. The bands $\left[\lambda_{2 l-1}(0), \lambda_{2 l-1}(\pi)\right]$ and $\left[\lambda_{2 l}(\pi), \lambda_{2 l}(0)\right]$ form the spectrum, precisely: $\sigma\left(-\frac{d^{2}}{d x^{2}}+\mu \cos x\right)=\bigcup_{l=0}^{\infty}\left[a_{l}, b_{l}\right]$ with $a_{l}=\left\{\begin{array}{l}\lambda_{l}(0) \text { if } l \text { is odd } \\ \lambda_{l}(\pi) \text { if } l \text { is even }\end{array} ; b_{l}=\left\{\begin{array}{l}\lambda_{l}(\pi) \text { if } l \text { is odd } \\ \lambda_{l}(0) \text { if } l \text { is even }\end{array}\right.\right.$. $-\frac{d^{2}}{d x^{2}}+\mu \cos x$ has no eigenvalues, it has purely essential spectrum. It is also shown by Reed and Simon (Volume 4, 1978) that for all $l \in \mathbb{N}, a_{l+1} \neq b_{l}$, so every gap occurs.

\subsection{Stark Potentials}

The Hamiltonian describing a quantum mechanical particle in a constant electric field $k \in \mathbb{R}^{n}$ is given by $P(k)=-\Delta+x . k$, where $V(x)=x . k$ is the Stark potential for the constant electric field $k$. The self-adjoint extension of $P(k)$ on $L^{2}\left(\mathbb{R}^{n}\right)$ with its natural domain $D(P(k))=$ $H^{2}\left(\mathbb{R}^{n}\right) \cap\left\{\varphi:(x . k) \varphi(x) \in L^{2}\left(\mathbb{R}^{n}\right)\right\}$ is called the Stark-Hamiltonian. We can consider here only the case of one-dimensional electric field. In fact, for example low-dimensional hydrogenic systems are realized in nature in the form of electron-hole pairs ("excitons") in lowdimensional quantum structures such as quantum wells, quantum wires, and carbon nanotubes (Pedersen, 2007). The multi-dimensional case is easily exploited via the spectral properties of self-adjoint operators, in particular the physical case $n=3$.

3.18. Theorem: Let $P(k)=-\Delta+x . k, k \in \mathbb{R}^{n} \backslash\{0\}$, be the Stark-Hamiltonian in $L^{2}\left(\mathbb{R}^{n}\right)$. Then, $\sigma(P(k))=\sigma_{\text {ess }}(P(k))=\mathbb{R}$, and the operator $P$ does not have eigenvalues. 
Proof: First note that $\sigma(P(k)) \neq \varnothing$. Otherwise the associated spectral family $E(\lambda)$ of $P(k)$ would be constant which would be in contradiction with $\lim _{\lambda \rightarrow+\infty} E(\lambda)=I$ and $\lim _{\lambda \rightarrow-\infty} E(\lambda)=0$. As $\sigma(P(k)) \subseteq \mathbb{R}$, let $\lambda \in \sigma(P(k))$ and some $\eta \in \mathbb{R}$. We want to show that $\eta \in \sigma(P(k))$.

Consider, for $\varphi \in L^{2}\left(\mathbb{R}^{n}\right):\left(U_{t} \varphi\right)(x)=\varphi(x-t k), t \in \mathbb{R}, x \in \mathbb{R}^{n}$ the group of translations on functions of $L^{2}\left(\mathbb{R}^{n}\right) .\left\{U_{t}: t \in \mathbb{R}\right\}$ is a family of unitary operators, thus $U_{t}(D(P(k)))=D(P(k))$ and $\sigma(P(k))=\sigma\left(U_{t} P(k) U_{t}\right)$, for all $t \in \mathbb{R}$.

Furthermore, for $\varphi \in D(P(k))$, we have: $U_{t} P(k) U_{-t} \varphi(x)=U_{t}(-\Delta+x \cdot k) \varphi(x+t k)=U_{t}(-\Delta \varphi)(x+t k)+U_{t}((x . k) \varphi(x+t k))=$ $(-\Delta \varphi)(x)+((x-t k) \cdot k) \varphi(x)=P(k) \varphi(x)-t|k|^{2} \varphi(x), t \in \mathbb{R}$, where $|k|^{2}=k_{1}^{2}+\cdots+k_{n}^{2}$ if $k=\left(k_{1}, \ldots, k_{n}\right)$. So, $U_{t} P(k) U_{-t}=P(k)-t|k|^{2}$, for all $t \in \mathbb{R}$. Let's take $=\frac{\lambda-\eta}{|k|^{2}}$, then: $\eta=$ $\lambda-t|k|^{2} \in \sigma(P(k))-t|k|^{2}=\sigma\left(P(k)-t|k|^{2}\right)=\sigma\left(U_{t} P(k) U_{-t}\right)=\sigma(P(k))$, thanks to spectral mapping theorem. We deduce that $\sigma(P(k))=\mathbb{R}$.

Now, if we assume that $\lambda$ is an eigenvalue of $P(k)$ with eigenvector $\varphi \in D(P(k)), \varphi \neq$ 0 , then $P(k) U_{-t} \varphi(x)=P(k) \varphi(x+t k)=\lambda \varphi(x+t k)=\lambda U_{-t} \varphi(x)$, and for all $t \in$ $\mathbb{R}, U_{t} P(k) U_{-t} \varphi(x)=\left(P(k)-t|k|^{2}\right) \varphi(x)=\lambda \varphi(x)$, so, $P(k) \varphi(x)=\left(\lambda+t|k|^{2}\right) \varphi(x)$.

We find that $\lambda+t|k|^{2}$ is also an eigenvalue of $P(k)$ for any $k \in \mathbb{R}^{n} \backslash\{0\}$. This yields an over-countable orthonormal system of $L^{2}\left(\mathbb{R}^{n}\right)$ which contradicts the fact that $L^{2}\left(\mathbb{R}^{n}\right)$ is separable. Therefore, $\sigma(P(k))=\sigma_{\text {ess }}(P(k))=\mathbb{R}$.

\section{RESONANCES OFSCHRÖDINGER OPERATORS}

Recently, substantial progress has been given in the analysis of the Schrödinger operator with perturbations going to 0 or $\infty$ as $|x| \rightarrow \infty$, or with periodic perturbations, and the works concern the calculation of spectra or resonances of these operators. We now examine the resonances of multi-dimensional Schrödinger operators and we apply our results to those created by the Schrödinger operator for the hydrogen atom.

\subsection{General Framework}

We have studied the discrete and essential spectrum of a self-adjoint operator $P=-\Delta+V$ on $L^{2}\left(\mathbb{R}^{n}\right)$, according to different behavior of $V$. The quantum resonances of $P$ appear as the poles of a meromorphic continuation of the resolvent of $P$, they also constitute the discrete spectrum of a spectral deformation family $P_{\mu}$ of $P$. In this section, we just recall the basic definitions and results and we sketch briefly the mathematical theory of resonances and apply it to the analysis 
of the important physical phenomenon of hydrogen atom (Aguilar and Combes, 1971; Balslev and Combes, 1971; Messirdi, 1994). Let $U_{\mu}, \mu \in \mathbb{R}$, be the one-parameter group of dilatations:

$$
\left(U_{\mu} \varphi\right)(x)=e^{n \mu / 2} \varphi\left(e^{\mu} x\right), \quad \varphi \in L^{2}\left(\mathbb{R}^{n}\right) .
$$

It is easy to check that $\left\{U_{\mu}: \mu \in \mathbb{R}\right\}$ forms a one-parameter unitary group and that $U_{\mu}(D(-\Delta))=H^{2}\left(\mathbb{R}^{n}\right)$ for all $\mu \in \mathbb{R}$. Thus, $U_{\mu}(-\Delta) U_{\mu}^{-1}=-e^{-2 \mu} \Delta$ is well defined for real $\mu$, and can be analytically continued into regions of complex $\mu$. Note that if $\operatorname{Im} \mu>0$, the spectrum of $U_{\mu}(-\Delta) U_{\mu}^{-1}$ is $e^{-2 \operatorname{Im} \mu}[0+\infty$ [, which is the positive half-axis rotated from the origin in the complex plane by an angle equal to $(-2 \operatorname{Im} \mu)$. On the other hand,

$$
P_{\mu}=U_{\mu} P U_{\mu}^{-1}=-e^{-2 \mu} \Delta+V\left(e^{\mu} x\right) .
$$

So, we want to consider a class of analytically continued potentials $V$ on a complex strip such that the same is true for the essential spectrum. Assume that $V$ is real and the family $\mu \rightarrow$ $V\left(x e^{\mu}\right)$ has an analytic continuation into a complex disk $\{|\mu|<\varepsilon\}$ as operators from the Sobolev space $H^{2}\left(\mathbb{R}^{n}\right)$ to $L^{2}\left(\mathbb{R}^{n}\right), V$ is called a dilation analytic potential. Thus, $\left(P_{\mu}\right)_{\mu}$ is, in this case, a family of non-self-adjoint analytic operators where $\mu$ runs in the disk $\{|\mu|<\varepsilon\}$. Assume also that $V(-\Delta+I)^{-1}$ is compact operator, then by Weyl Theorem:

$$
\sigma_{e s s}\left(P_{\mu}\right)=e^{-2 \mu} \sigma_{e s s}\left(-\Delta+e^{2 \mu} V\left(e^{\mu} x\right)\right)=e^{-2 \mu} \sigma_{e s s}(-\Delta)=e^{-2 \mu}[0,+\infty[.
$$

As an example of the above considerations we have the potential $V(x)=\frac{-\gamma}{|x|}, \gamma>0$, of the hydrogen atom Hamiltonian $P_{h}=-\Delta-\frac{\gamma}{|x|}$. A complex number $\lambda$ is a resonance of $P$ if $\operatorname{Re} \lambda>\inf \sigma_{e s s}(P)$ and if there exists $\mu$ small enough, $|\mu|<\varepsilon$, such that $\lambda \in \sigma_{\text {disc }}\left(P_{\mu}\right)$. It is well known that the resolvent operator $(P-z)^{-1}, z \in \mathbb{C} \backslash \mathbb{R}$, is a meromorphic function and its poles are precisely the resonances of $P$ (Messirdi et al., 2018). Furthermore, the complex poles of $(P-z)^{-1}$ coincide with the complex poles of the scattering matrix of the system which are interpreted as resonances (Belmouhoub and Messirdi, 2017).

4.1. Example: The method of complex scaling allows to compute the complex poles or resonances of hydrogen atom Hamiltonian $P_{h}=-\Delta-\frac{\gamma}{|x|}, \gamma>0$.

$$
P_{h, \mu}=U_{\mu} P_{h} U_{\mu}^{-1}=-e^{-2 \mu} \Delta-e^{-\mu} \frac{\gamma}{|x|}, \mu \in \mathbb{R} .
$$

Let $e_{h, l}=-\frac{1}{4 l^{2}}, l \in \mathbb{N}^{*}$, be the eigenvalues of the hydrogen atom. Then the spectrum of $P_{h, \mu}$ is: $\quad \sigma_{e s s}\left(P_{h, \mu}\right)=\sigma_{e s s}\left(-e^{-2 \mu} \Delta-e^{-\mu} \frac{\gamma}{|x|}\right)=\sigma_{e s s}\left(-e^{-2 \mu} \Delta\right)=e^{-2 \operatorname{Im} \mu}[0,+\infty[$,

$$
\sigma\left(P_{h, \mu}\right)=\left\{-\frac{1}{4 l^{2}}: l \in \mathbb{N}^{*}\right\} \cup e^{-2 \operatorname{Im} \mu}[0,+\infty[.
$$


The real eigenvalues of $P_{h, \mu}$ coincide with eigenvalues of $P_{h}$, complex eigenvalues (resonances) of $P_{h, \mu}$ lie in the complex half-plane $\{\operatorname{Imz}<0: z \in \mathbb{C}\}$ and are locally independent of $\mu$ (see Messirdi et al., 2018).

\subsection{Resonances for Periodic Schrödinger Operators}

We review some results about the resonances for a periodic Schrödinger operator $P=-\Delta+V$ on $L^{2}\left(\mathbb{R}^{n}\right)$. If $V$ is a real multiplicative potential and periodic with respect to some lattice $\Gamma$ in $\mathbb{R}^{n}$, it was stated in the previous section that the spectrum of $P$ consists of bands, these bands consist of purely continuous spectrum. In a neighborhood of such a band, the resonances of $P$ are defined and studied by analytic extension of the resolvent $(P-z)^{-1}$. It has been shown by Gérard (1990) that $(P-z)^{-1}$ extends across the spectrum of $P$ to the complementary of a discrete set of points, called Van Hove singularities in solid state physics. The existence of such an extension is interesting in the solid state physics to introduce the resonances of the studied system, nevertheless it is mathematically quite difficult to realize. In particular, the singularities of $(P-z)^{-1}$ are different when we consider the local extension of $(P-z)^{-1}$ in a small neighborhood of an energy level $z_{0} \in \sigma(P)$, and when we consider the global extension of $(P-$ $z)^{-1}$ to a bounded open set $U$ in $\mathbb{C}$. We will assume that $V$ is $\Delta$-bounded with relative bound strictly less than 1 , so that $P$ is self-adjoint with domain $H^{2}\left(\mathbb{R}^{n}\right)$. Using the same notations of the section 3.4, $H=L^{2}\left(\Omega^{*}, d \theta ; L^{2}(\Omega)\right)=\int_{\Omega^{*}}^{\oplus} H_{\theta} d \theta$, and $\int_{\Omega^{*}}^{\oplus} P_{\theta} d \theta$, where $P_{\theta}$ is the restriction of $P$ to $H_{\theta}=L^{2}(\Omega) .(P-z)^{-1}$ is given by $(P-z)^{-1} \varphi=(2 \pi)^{-n / 2} \int_{\Omega^{*}}^{\oplus \frac{M(\theta, z)}{f(\theta, z)}} d \theta$ where $f(\theta, z)$ and $M(\theta, z)$ are holomorphic for $\theta \in W$ a bounded set in $\mathbb{C}^{n}$ such that $\Omega^{*} \subset W$, and $z \in \mathbb{C}$, as bounded operators on $L^{2}(\Omega)$.

Furthermore, $f\left(\theta+\gamma^{*}, z\right)=f(\theta, z)$ and $M\left(\theta+\gamma^{*}, z\right)=M(\theta, z)$ for all $\gamma^{*} \in \Gamma, \theta \in$ $W$ and $z \in \mathbb{C}$. Now to extend $(P-z)^{-1}$ as a meromorphic function is a well-known problem in complex analysis (Bros and Pesenti, 1983). The poles of this extension (Van Hove singularities) are effectively the resonances of the operator $P$.

\section{CONCLUSIONS}

The main object of this paper is to study the spectrum of several classes of Schrödinger operators and to look at some important examples occurring in mathematical physics (e.g. the harmonic oscillator, the Stark effect, and the hydrogen atom). We also examine the resonances of multi-dimensional Schrödinger operators and we apply our results to periodic potentials and 
hydrogen atom. In the proofs of our main results we make use some algebraic and spectral considerations.

\section{ACKNOWLEDGEMENTS}

The authors would like to thank the referees for their valuable comments which helped to improve the manuscript.

\section{CONFLICT OF INTERESTS}

There is no conflict of interests.

\section{REFERENCE}

Aguilar, J \& Combes, J.M. 1971. A class of analytic perturbations for one-body Schrödinger Hamiltonians. Communications in Mathematical Physics, 22: 269-279.

Balslev, E \& Combes, J.M. 1971. Spectral properties of many-body Schrödinger operators with dilatation-analytic interactions. Communications in Mathematical Physics, 22: 280-294.

Belmouhoub, S \& Messirdi, B. 2017. Singular Schrödinger Operators via Grushin Problem Method. Analele Universitatii din Oradea, Fascicola Matematica, 24(1): 83-91.

Bros, J \& Pesenti, D. 1983. Fredholm resolvents of meromorphic kernels with complex parameters: a Landau singularity and the associated equations of type $U$ in a non holonomic case. Journal des Mathématiques Pures et Appliquées, 62(9): 215-252.

Brüning, J. 1989. On Schrödinger operators with discrete spectrum. Journal of Functional Analysis, 85(1): 117-150.

Cycon, H.L., Froese, R.G., Kirsch, W \& Simon, B. 1987. Schrödinger Operators with Applications to Quantum Mechanics and Global Geometry. ISBN: 10: 0387167587, Springer-Verlag Berlin Heidelberg, pp.160-171.

Friedrichs, K. 1934. Spektraltheorie halbbeschrankter Operatoren und Anwendung auf die Spektralzerlegung von Differential operatoren. Mathematische Annalen, 109: 465-487.

Gérard, C. 1990. Resonance theory for periodic Schrödinger operators. Bulletin de la Société Mathématique de France, 118: 27-54.

Gherib, F \& Messirdi, B. 2005. Spectral classification in a periodic structure. Lecturas Matematicas, 26: 145-163.

Landau, L.D \& Lifshitz, E.M. 1977. Theoretical Physics 3. Quantum Mechanics: NonRelativistic Theory. ISBN:13: 978-0080291406, Pergamon, pp. 256-259. 
Messirdi, B. 1994. Asymptotique de Born Oppenheimer pour la prédissociation moléculaire (cas de potentiels réguliers). Annales de l'Institut Henri Poincaré, A. 61(3): 255-292.

Messirdi, B., Messirdi, S \& Messirdi, M. 2018. Complex symmetric operators and semiclassical resonances. Asian-European Journal of Mathematics, 11(1): DOI: 10.1142/S1793557118500444.

Muminov, M.I \& Shodiev, U.R. 2011. On the essential spectrum of a four-particle Schrödinger operator on alattice. Siberian Advances in Mathematics, 21(4): 292.303.

Pedersen, T.G. 2007. Exact polarizability of low-dimensional excitons. Solid State Communications, 141: 569.

Persson, A. 1960. Bounds for the discrete part of the spectrum of a semi-bounded Schrodinger operator. Mathematica Scandinavica, 8: 143-153.

Reed, M. \& Simon, B. 1978. Methods of Modern Mathematical Physics: Analysis of Operators. $1^{\text {st }}$ edition, Volume 4, Academic Press, pp. 55-60, Hardcover ISBN: 9780125850049, eBook ISBN: 9780080570457, 325p.

Reed, M. \& Simon, B. 1979. Methods of Modern Mathematical Physics: Scattering Theory. $1^{\text {st }}$ edition, Volume 3, Academic Press, pp. 144-147, Hardcover ISBN: 978-0-12-5850032; eBook ISBN: 978-0-08-092538-7, 468p.

Simon, B. 2009. Schrödinger operators with purely discrete spectrum. Methods of Functional Analysis and Topology, 15(1): 61-66. 